\title{
Pedigree analysis in White Shorthaired goat: First results
}

\author{
Marta Oravcová \\ Animal Production Research Centre Nitra, Lužianky, Slovakia
}

\begin{abstract}
Pedigree records of 1682 animals of the White Shorthaired goat in Slovakia were investigated. The reference population was defined as the animals born from 2008 to 2011 with at least one ancestor known in the second ancestral generation (670 animals kept in eight flocks). The numbers of founders (286), ancestors (256), effective founders (73), effective ancestors (45) and founder genome equivalents (32) were assessed. Fifteen ancestors were needed to explain $50 \%$ of genetic variability. Marginal contributions of the ten most influential ancestors varied between $5.45 \%$ and $2.47 \%$ and accounted for $39.8 \%$ of genetic variability. The mean values of inbreeding and co-ancestry were $0.69 \%$ and $1.55 \%$, respectively. The effective population size was assessed to consist of 182 and 142 individuals, depending whether it was calculated from the individual increase in inbreeding or the individual increase in co-ancestry. The number of maximum generations traced, fully traced generations and equivalent complete generations traced were 5.62, 1.97 and 3.04, respectively. The first, second and third ancestral generation were $100 \%, 83 \%$ and $71 \%$ complete, respectively. The completeness decreased to as low as $35 \%$ and $11 \%$ in the fourth and fifth generation. To be able to keep genetic links across generations in touch, the amount of pedigree information needs to be increased. This is a serious requirement for appropriate monitoring and management of genetic relations within the population.
\end{abstract}

Keywords: goats, genealogical information, founders, ancestors, founder genome equivalents, inbreeding, co-ancestry, effective population size

Archiv Tierzucht 56 (2013) 53, 547-554

doi: $10.7482 / 0003-9438-56-053$

Corresponding author:

Marta Oravcová; email: oravcova@cvzv.sk

Animal Production Research Centre Nitra, Hlohovecká 2, 95141 Lužianky, Slovak Republic

(C) 2013 by the author; licensee Leibniz Institute for Farm Animal Biology (FBN), Dummerstorf, Germany. This is an Open Access article distributed under the terms and conditions of the Creative Commons Attribution 3.0 License (http://creativecommons.org/licenses/by/3.0/).

Received: 24 October 2012

Accepted: 1 March 2013 Online: 26 April 2013

(htp.//creativecommons.org/licenses/by/3.0/). 


\section{Introduction}

The national goat population in Slovakia amounts to about 30000 heads. The White Shorthaired breed predominates. Goat-farming is of minor importance and has features of self-subsistence. An extensive production system with one kidding per year is applied. In performance testing a few flocks of the White Shorthaired breed are included. Selection is performed under the guidance of the Sheep and Goats Breeders' Association and the Breeding Services of Slovakia. The recorded population involves 700 to 800 females at present. Breeding strategies are aimed at improvement of milk (selection on phenotypic values), prolificacy and exterior (selection on linear scores using a three-point and a twenty-point scale, respectively) and have an impact on evolution of the breed. These should take genetic diversity aspects into account since genetic diversity is the basis for genetic adaptability for all changes over time such as changes in climate, pollution and diseases (Baldursdóttir 2010). Information on inbreeding and co-ancestry is important when effort to prevent breeds from extinction is in place (Goyache at al. 2003, Gutiérrez et al. 2005). Information on probabilities of gene origin (founders, ancestors, effective founders, effective ancestors and founder genome equivalents) enables to provide an insight into the genetic history of the breed. A tool for assessment of these parameters is the pedigree analysis. With populations of small ruminants in Slovakia, it was recently completed with the Valachian breed (Oravcová \& Krupa 2011, Oravcová \& Margetín 2011). The genetic variability of goats has not been studied until now.

The objective of this study was to assess the parameters derived from probabilities of gene origin and identity-by-descent probabilities in a population of the White Shorthaired breed. The pedigree completeness was analysed.

\section{Material and methods}

Genealogical information on 1682 animals (1 485 females and 197 males) of the White Shorthaired goat was investigated. In total, 971 animals with progeny and 711 animals without progeny were considered. For males, 136 sires and 95 grandsires were identified. For females, 835 dams and 503 grand-dams were identified. Due to only natural mating being applied, the ratio dams/sires in a pedigree was found to be low. The average number of descendants per sire was 10.2. The average number of descendants per dam was 1.6. Up to $70 \%$ of sires were individuals with 1 to 10 descendants. Less than $10 \%$ of sires had 40 and more descendants. No dams with more than 10 descendants were found. Up to $80 \%$ of all animals in the pedigree were animals with both parents known and $16 \%$ were animals with no parents known. The proportion of animals with one parent known was $4 \%$.

To assess probabilities of gene origin and identity-by-descent probabilities, the population under study (reference population) was defined as the animals born from 2008 to 2011. Only animals with at least one ancestor known in the second ancestral generation were taken into account ( 670 individuals in eight flocks). Of these, 625 were females and 45 were males. Pedigree records were provided by the Breeding Services of Slovakia. The number of founders, the effective number of founders, the effective number of ancestors and the number of ancestors explaining $50 \%$ and $75 \%$ of genetic variability, as well as the founder genome equivalents were calculated. The mean values of coefficients of inbreeding and co- 
ancestry were calculated by averaging respective values of all individuals in the reference population. The realized effective size was calculated taking into account (a) the individual increase in inbreeding and (b) the individual increase in co-ancestry. The programme ENDOG v.4.8 (Gutiérrez \& Goyache 2005) and the SAS software (2009; SAS Institute Inc., Cary, NC, USA) were used for analyses.

The pedigree completeness was evaluated by the index of pedigree completeness (MacCluer et al. 1983) taking into account contributions from the maternal and paternal lines. In addition, the number of maximum generations traced, fully traced generations and equivalent complete generations traced (Maignel et al. 1996, Gutiérrez \& Goyache 2005) were calculated. The number of fully traced generations was defined as generations separating the offspring from the furthest generation where $2^{\mathrm{g}}$ ancestors are known (generation 0 are ancestors with unknown parents). The number of maximum generations traced was defined as generations separating the offspring from the furthest ancestor. The number of equivalent complete generations traced was defined as the sum of $(1 / 2)^{n}$ where $n$ is the number of generations separating the offspring from each known ancestor (parent=1, grandparent=2 etc.).

Among parameters of probabilities of gene origin, the number of founders $(f)$ was defined as animals with one or two unknown parents. The effective number of founders $\left(f_{e}\right)$ was defined as the number of equally contributing founders that would produce the same genetic diversity in the population under study (Lacy 1989) and was calculated as

$$
f_{e}=1 / \sum_{k=1}^{f} q_{k}^{2}
$$

where $q_{k}$ is the expected proportional genetic contribution of founder $k$. The ancestors (founders or not) were identified using the method proposed by Boichard et al. (1997). The effective number of ancestors $(f a)$ was defined as the minimum number of ancestors explaining the genetic diversity of the population under study (Boichard et al. 1997) and was calculated as

$$
f_{a}=1 / \sum_{j=1}^{\mathrm{a}} q_{j}
$$

where $q_{j}$ is the marginal contribution of ancestor $j$ not explained by any other ancestor chosen before (contribution independent of the contributions of the other ancestors). The number of founder genome equivalents $\left(f_{g}\right)$ was defined as the number of founders producing the same genetic diversity in the population under study if the founders were equally represented and no loss of alleles occurred (Lacy 1989). The number of founder genome equivalents was obtained by the inverse of twice the average co-ancestry of the individuals in the reference population (Caballero \& Torro 2000).

Among parameters of identity-by-descent probabilities, the coefficient of co-ancestry was defined as the probability that any two alleles sampled at random (one from each individual) are identical copies of an ancestral allele (Malécot 1948 as cited by Ghafouri-Kesbi 2010). The individual coefficient of inbreeding was defined as the probability that an individual has two identical alleles by descent and was calculated following Meuwissen \& Luo (1992). The coefficient of individual increase in inbreeding (Gutiérrez \& Goyache 2005) was calculated 
from the individual coefficient of inbreeding $F_{i}$ taking the number of equivalent complete generations t into account:

$$
\Delta F_{i}=1-\sqrt[t-1]{1-F_{i}}
$$

The coefficient of individual increase in co-ancestry between any pair of individual $j$ and individual $k$ was calculated as

$$
\Delta c_{j k}=1-\frac{g j+g k}{2} \sqrt{1-c_{j k}}
$$

where $c_{j k}$ is the inbreeding of a descendant from both and $g_{j}$ and $g_{k}$ are the equivalent complete generations for the parents (Cervantes et al., 2011). By averaging the individual increase in inbreeding

$$
\overline{N_{e}}=\frac{1}{2 \Delta \bar{F}}
$$

(Guttiérrez et al. 2008) and the individual increase in co-ancestry

$$
\overline{N_{e c}}=\frac{1}{2 \Delta \bar{c}}
$$

(Cervantes et al. 2011), the realized effective population size can be estimated.

\section{Results and discussion}

Table 1 shows the following indicators of the pedigree completeness level assessed for the reference population of White Shorthaired goat (animals born from 2008 to 2011 with at least one ancestor known in the second ancestral generation): 5.62 maximum generations traced, 1.97 fully traced generations and 3.04 equivalent complete generations. The first, second and third ancestral generation was $100 \%, 83 \%$ and $71 \%$ complete, respectively. The completeness decreased to as low as $35 \%$ and $11 \%$ in the fourth and fifth generation. No more than $3 \%$ completeness was found from the sixth to ninth generation. The large proportion of missing parents in a pedigree may cause underestimation of inbreeding (Boichard et al. 1997). Therefore, limited knowledge of pedigree should be taken into account when looking at the results of pedigree analysis in White Shorthaired goat. To the best knowledge of this study's author, only a single study has investigated the pedigree completeness in goats (Baldursdóttir 2010). With genealogical information of 2240 Icelandic goat individuals, the pedigree completeness in the fifth generation was found to be between $25 \%$ and $39 \%$ for the youngest animals. Comparisons with Valachian sheep (Oravcová \& Krupa 2011) and Improved Valachian and Tsigai sheep (unpublished data) showed more complete pedigree records in Slovakian sheep than in goats (3.71 and 5 vs. 3.04 equivalent complete generations). In contrast, pedigree quality of Zandi sheep (3.15 equivalent complete generations) reported by Ghafouri-Kesbi (2010) was similar to pedigree quality found in White Shorthaired goat. Huby et al. (2003) reported 4.1 to 6.7 equivalent complete generations with exception of one breed. Moreover, Danchin-Burge et al. (2010) reported 4.6 to 10.5 equivalent complete generations in various French sheep breeds. 
The parameters of probabilities of gene origin derived for the reference population of White Shorthaired goat are given in Table 1. The number of founders and the effective number of founders were 286 and 73, respectively. The more balanced the expected contributions of founders, the higher the effective number of founders. Consequently, the smaller number of effective founders compared to the number of founders indicates the excessive use of some animals as parents or a disequilibrium between the founder contributions (Goyache et al. 2003, Ghafouri-Kesbi 2010). The ratio $f_{e} / f$ was 0.26 and was almost the same as the ratio $f_{e} / f(0.25)$ in Xalda sheep (Goyache et al. 2003) with genealogical information of 561 animals investigated ( 329 founders and 81 effective founders). In contrast, the ratio $f_{e} / f$ was 0.14 in Zandi sheep (Ghafouri-Kesbi 2010) with genealogical information of 2566 animals investigated (615 founders and 86 effective founders).

The number of ancestors contributing to the reference population of White Shorthaired goat was assessed 256 and was about $90 \%$ of the founder animals (vs. $72 \%$ and $97 \%$ in Xalda and Zandi sheep, respectively). This relatively high number of ancestors is typical for populations lacking long historical pedigrees by showing a short period of time between the youngest generations and the founders (Gutiérrez et al. 2003). The effective number of ancestors, defined as the minimum number of ancestors necessary to explain the complete genetic diversity of a population (considering the contribution of an ancestor not yet accounted for by other ancestors), was smaller (45) and was $16 \%$ of the founder animals. A small effective number of ancestors accounts for the loss of genetic variability from parent to offspring due to bottleneck (Álvarez et al. 2010) and is a result of the fact that selection increases representation of some animals in a pedigree (Goyache et al. 2003). In White Shorthaired goat, selection in the early 1990s allowed only pulled animals to be accepted for breeding. The ratio $f_{e} / f_{a}$ in White Shorthaired goat was 1.6. The higher the value of this ratio, the higher the bottleneck effect resulting from the decrease of the number of reproducers over the generations (de Oliviera et al. 2012). When the ratio $f_{e} / f_{a}$ is close to unity, the population is stable in terms of effectively contributing ancestors and the bottleneck has not played a significant role in the population (Fair et al. 2012). The effective number of ancestors in White Shorthaired goat was similar to that in Xalda sheep (40 effective ancestors) and the ratio $f_{e} / f_{a}$ was smaller than that reported by Goyache et al. (2003) for Xalda sheep (1.6 vs. 2).

The calculation of $f_{a}$ involves assessment of marginal contribution of each ancestor to the genetic variability of the reference population starting from the contribution of the most influential ancestor (Ghafouri-Kesbi 2010). Marginal contributions of the ten most influential ancestors varied between $5.45 \%$ and $2.47 \%$ and accounted for $39.8 \%$ of the genetic variability of the reference population of White Shorthaired goat. The most influential ancestors were nine males with 69 to 20 descendants per buck and one female with nine descendants. Fifty percent of the genetic variability of the reference population was explained by marginal contributions of 15 ancestors. Seventy-five percent of the genetic variability of the reference population was explained by marginal contributions of 43 ancestors (Table 1).

In contrast to the effective number of ancestors, the number of founder genome equivalents accounts for all random losses of genes during segregations and consequently provides a smaller number than $f_{e}$ and $f_{a}$ (Boichard et al. 1997). It was assessed 32 for the reference population of White Shorthaired goat (Table 1). 
Table 1

Parameters of pedigree completeness, probabilities of gene origin and identity-by-descent probabilities

\begin{tabular}{|c|c|}
\hline Parameter & Reference population \\
\hline No. of maximum generations, mean value & 5.62 \\
\hline No. of fully traced generations, mean value & 1.97 \\
\hline No. of equivalent complete generations, mean value & 3.04 \\
\hline Pedigree completeness in 1st generation of ancestors & 100 \\
\hline Pedigree completeness in 2nd generation of ancestors & 83 \\
\hline Pedigree completeness in 3rd generation of ancestors & 71 \\
\hline Pedigree completeness in 4th generation of ancestors & 35 \\
\hline Pedigree completeness in 5 th generation of ancestors & 11 \\
\hline Pedigree completeness in 6th to 9th generation of ancestors & $\leq 3$ \\
\hline No. of founder animals & 286 \\
\hline No. of ancestors & 256 \\
\hline Effective No. of founders $\left(f_{e}\right)$ & 73 \\
\hline $\begin{array}{l}\text { Effective No. of ancestors }\left(f_{a}\right) \\
N A_{50}\end{array}$ & $\begin{array}{l}45 \\
15\end{array}$ \\
\hline $\mathrm{NA}_{75}$ & 43 \\
\hline Founder genome equivalents $\left(f_{g}\right)$ & 32 \\
\hline Inbreeding, mean value ( $\bar{F} \%)$ & 0.69 \\
\hline Co-ancestry, mean value ( $\bar{c} \%)$ & 1.55 \\
\hline$\overline{N_{e}}$ (from increase in inbreeding) & 182 \\
\hline$\overline{N_{e c}}$ (from increase in co-ancestry) & 142 \\
\hline
\end{tabular}

$\mathrm{NA}_{50}-\mathrm{No}$. of ancestors explaining $50 \%$ of genetic variability, $\mathrm{NA}_{75}-$ No. of ancestors explaining $75 \%$ of genetic variability, $N_{e}, N_{e c}$ - Effective population size

The parameters of identity-by-descent probabilities derived for the reference population of White Shorthaired goat are shown in Table 1. The mean values of coefficients of inbreeding and co-ancestry were assessed $0.69 \%$ and $1.55 \%$, respectively. In total, $0.30 \%$ matings between half sibs and $0.24 \%$ matings between parent-offspring were identified. The low inbreeding level may be a result of insufficient pedigree completeness as well as a result of the breeders' intention to avoid mating of related animals. In contrast, the mean values of coefficients of inbreeding and co-ancestry in Zandi sheep assessed at $1.46 \%$ and $1.21 \%$ were reported by Ghafouri-Kesbi (2010). The co-ancestry describes the amount of relations among individuals in a breeding programme and the effectiveness of a selection programme. The opposite of co-ancestry may be considered as diversity according to Ghafouri-Kesbi (2010). The higher the co-ancestry, the lower is the genetic diversity. For effective selection, the coancestry needs to be managed.

The parameters $\overline{N_{e}}$ and $\overline{N_{e c}}$ (effective population size assessed from the individual increase in inbreeding and the individual increase in co-ancestry, respectively) are assumed to be the measure of the same cumulated drift processes in a population (Cervantes et al. 2011). A difference between $\overline{N_{e}}$ and $\overline{N_{e c}}$ would characterize the influence of preferential matings in a 
population. It is not easy to interpret a difference between $\overline{N_{e}}$ and $\overline{N_{e c}}$ found in the population of White Shorthaired goat because similar values of $\overline{N_{e}}$ and $\overline{N_{e c}}$ may result from a shallow pedigree. The value of $\overline{N_{e c}}$ was found to be smaller than the value of $\overline{N_{e}}(142 \mathrm{vs}$. 182) which is in contrast to findings of Cervantes et al. (2011) who referred opposite relationship between $\overline{N_{e}}$ and $\overline{N_{e c}}$ in Spanish horse breeds.

No clear relationship between the results provided from calculations of parameters of probabilities of gene origin and parameters of identity-by-descent probabilities in White Shorthaired goat can be found. The parameters of probabilities of gene origin indicated a decrease in genetic diversity between the founders and the reference population (comparisons between founders and ancestors and between effective founders and effective ancestors as well as founder genome equivalents). This situation, however, is typical for populations lacking long historical pedigrees by showing a short period of time between the youngest generations and the founders (Gutiérrez et al. 2003). No genetic diversity issues were indicated by the values of effective population size. Findings presented in this study need to be interpreted with caution due to a limited history of a pedigree. Incomplete pedigree records with only one third and one tenth of ancestors known in the fourth and fifth ancestral generation may cause underestimation of inbreeding and increase in inbreeding as well (according to Cervantes et al. 2008, increase in inbreeding tends to stabilize with about five equivalent complete generations). The high number of the founders and ancestors and the disequilibrium between effective founders and effective ancestors may, in addition to preferential use of some animals and weak bottleneck effects, also be due to insufficient pedigree information.

In conclusion, the study shows the first results of a pedigree analysis in White Shorthaired goat in Slovakia. There were found small numbers of effective founders, effective ancestors and founder genome equivalents (in comparison to founders and ancestors), whereas the values of effective population size were assessed relatively high. Starting with the fourth generation of ancestors, the pedigree completeness was extremely low. To be able to keep genetic links across generations in touch, the amount of pedigree information needs to be increased. This is a serious requirement for appropriate monitoring and management of genetic relations within the population.

\section{References}

Álvaréz I, Royo LJ, Pérez-Pardal L, Fernández I, Payeras L, Goyache F (2010) Assessing losses of genetic variability in the endangered Mallorquí horse. Czech J Anim Sci 55, 456-462

Baldursdóttir BK (2010) Genetic variation within the Icelandic goat breed. Assessment using population data and DNA analysis. MS-thesis, Agricultural University of Iceland, Faculty of Land and Animal Resources

Boichard D, Maignel L, Verrier E (1997) The value of using probabilities of gene origin to measure genetic variability in a population. Genet Sel Evol 29, 5-23

Caballero A, Toro MA (2000) Interrelations between effective population size and other pedigree tools for the management of conserved populations. Genet Res 75, 331-343

Cervantes I, Goyache F, Molina A, Valera M, Gutiérrez JP (2008) Application of individual increase in inbreeding to estime realized effective sizes from real pedigrees. J Anim Breed Genet 125, 301-310

Cervantes I, Goyache F, Molina A, Valera M, Gutiérrez JP (2011) Estimation of effective population size from the rate of coancestry in pedigreed populations. J Anim Breed Genet 128, 56-63 
Danchin-Burge C, Palhière I, François D, Bibé B, Leroy G, Verrier E (2010) Pedigree analysis of seven small French sheep populations and implications for the management of rare breeds. J Anim Sci 88, 505-516

De Oliviera AP, Malhado CHM, Carneiro PLS, Filho RM, Silveira ÉS, Souza LA, Muniz LMS, Azevêdo DMMR (2012) Pedigree analysis on the population of Gir cattle in Northeast Brazil. Rev Bras Zootec 41, 1153-1157

Fair MD, van Wyk JB, Cloete SWP (2012) Pedigree analysis of an ostrich breeding flock. S Afr J Anim Sci 42, $114-122$

Ghafouri-Kesbi F (2010) Analysis of genetic diversity in a close population of Zandi sheep using genealogical information. J Genet 89, 479-483

Goyache F, Gutiérrez JP, Fernández I, Gomez E, Alvarez I, Diéz J, Royo LJ (2003) Using pedigree information to monitor genetic variability of endangered populations: the Xalda sheep breed of Asturias as an example. J Anim Breed Genet 120, 95-105

Gutiérrez JP, Altarriba J, Díaz C, Quintanilla R, Cañón J, Piedrafita J (2003) Pedigree analysis of eight Spanish beef cattle breeds. Genet Sel Evol 35, 43-63

Gutiérrez JP, Marmi J, Goyache F, Jordana J (2005) Pedigree information reveals moderate to high levels of inbreeding and a weak population structure in the endangered Catalonian donkey breed. J Anim Breed Genet 122, 378-386

Gutiérrez JP, Goyache F (2005) A note on ENDOG: a computer program for analysing pedigree information. J Anim Breed Genet 122, 172-176

Gutiérrez JP, Cervantes I, Molina A, Valera M, Goyache F (2008) Individual increase in inbreeding allows estimating effective sizes from pedigree. Genet Sel Evol 40, 359-378

Huby M, Griffon L, Moureax S, De Rochambeau H, Danchin-Burge C, Verrier É (2003) Genetic variability of six French meat sheep breeds in relation to their genetic management. Genet Sel Evol 35, 637-655

Lacy RC (1989) Analysis of founder representation in pedigrees: Founder equivalents and founder genome equivalents. Zoo Biol 8, 111-123

MacCluer JW, Boyce AJ, Dyke B, Weitkamp LR, Pfenning DW, Parsons CJ (1983) Inbreeding and pedigree structure in Standardbred horses. J Hered 74, 394-399

Maignel L, Boichard D, Verrier E (1996) Genetic variability of French dairy breeds estimated from pedigree information. Interbull Bull 14, 49-54

Meuwissen THE, Luo Z (1992) Computing inbreeding coefficients in large populations. Genet Sel Evol 24, 305313

Oravcová M, Krupa E (2011) Pedigree analysis of the former Valachian sheep. Slovak J Anim Sci 44, 6-12

Oravcová M, Margetín M (2011) Preliminary assessment of trends in inbreeding and average relatedness of the former Valachian sheep. Slovak J Anim Sci 44, 90-96

SAS (2009) SAS/STAT ${ }^{\oplus} 9.2$ User's Guide. 2nd ed., SAS Institute Inc., Cary, NC, USA 